\title{
Epitaxial strain energy measurements of GaN on sapphire by Raman spectroscopy
}

\author{
H. J. Park ${ }^{1}$, C. Park ${ }^{2}$, S. Yeo 2 , S.W. Kang ${ }^{1}$, M. Mastro ${ }^{1}$, O. Kryliouk ${ }^{1}$, and T. J. Anderson ${ }^{1 *}$ \\ ${ }^{1}$ Department of Chemical Engineering, University of Florida, Gainesville, FL 32611, USA \\ ${ }^{2}$ School of Chemical Engineering and Technology, Yeungnam University, Gyeongsan 712-749, Korea
}

Received 13 July 2004, revised 12 August 2004, accepted 27 January 2005

Published online 17 March 2005

PACS 61.72.Hh, 68.35.Gy, 68.60.Dv, 78.30.Fs, 81.05.Ea, 81.70.Fy

This study reports a non-destructive method of measuring the residual strain in the GaN epilayer grown on sapphire substrate by micro-Raman spectroscopy. Operating in confocal mode this method allows a depth-dependent measurement of residual strain in the epitaxial layer without prior treatment of the sample. This approach to measurement of residual strain is demonstrated on $\mathrm{GaN}$ epitaxial films grown by both MOCVD and H-MOVPE. In the case of MOCVD grown films, the biaxial strain energy was found to vary from 0 (GaN surface) to $5.0 \mathrm{~kJ} / \mathrm{mole}(\mathrm{GaN} / \mathrm{sapphire}$ interface), but in the case of H-MOVPE grown samples the strain energy varied from $6.5 \mathrm{~kJ} / \mathrm{mole}$ - hydrostatic strain (GaN surface) to 25.0 $\mathrm{kJ} /$ mole - biaxial strain (GaN/sapphire interface), indicating that the surface layer of the N-terminated $\mathrm{H}$ MOVPE material is not free from strain. Estimates are given for the curvature of substrate, lattice parameter of epitaxial layer, and the interface shear modulus.

(C) 2005 WILEY-VCH Verlag GmbH \& Co. KGaA, Weinheim

\section{Introduction}

There is considerable interest in the growth of high structural quality GaN epitaxial films for optoelectronic and electronic devices. GaN epitaxial layers grown on the commercially available substrates such as sapphire, however, contain residual strains produced by mismatch in the lattice constants and thermal expansion coefficients between the GaN film and substrate [1]. Sufficient residual strain in the film will induce formation of dislocations and stacking faults, which degrade device performance. Residual strain also leads to wafer bowing, making device processing more difficult. Thus a method to non-destructively measure residual strain is important.

Raman spectroscopy is well established as a non-destructive and relatively rapid method to determine the averaged values of residual strain. Strain energy profiles have been measured by using multiple samples of different thicknesses or probing a sample with variable absorption characteristics (e.g., variable excitation wavelength, confocal optics.) Given potential run-to-run variations in the sample strain state, the latter approach is preferred. The Raman shift of the $\mathrm{GaN} \mathrm{E}_{2}$ mode has been reported by several groups, and the reported values vary in the relatively large range 565 to $572 \mathrm{~cm}^{-1}[2,3]$, reflecting differences in the residual strain in the epilayer after growth and thus the sensitivity of the method. The reported shifts of the $\mathrm{E}_{2}$ mode for freestanding GaN samples, however, show the relatively wide range 566-568 $\mathrm{cm}^{-1}$ [2-5], suggesting that freestanding GaN films grown by HVPE techniques usually contain high impurity levels, resulting in hydrostatic stress. The $\mathrm{E}_{2}$ mode of strain-free GaN is known to be $567.6 \mathrm{~cm}^{-1}$ at room temperature [6]. The curvature of the substrate is also related to the strain in the film

"Corresponding author: e-mail: tim@ufl.edu, Phone: +1 352392 0946, Fax: +1 3523929673 
and is usually measured by laser interference or stylus profiling technique. Raman spectroscopy can be used as a versatile technique to measure the curvature of the substrate, and it provides a measurement that is insensitive to the surface roughness, as compared to the other techniques.

In this study, Raman spectroscopy was utilized to measure the residual strain in the epilayer, the curvature of the substrate, and the lattice parameters of the epilayer. Stoney's equation [7] was used to calculate the curvature of the substrates, and Shen's approach [8] was used to estimate lattice parameters and elastic compliances. Frank-van der Merwe's thick overgrowth model [9] was then applied to the MOCVD grown sample to check the validity of the assumption of isotropic strain by comparing the calculated shear modulus at the GaN/sapphire interface with those of the bulk crystals. Finally, the measured strain energy was added to the Gibbs energy of formation of GaN, and the lowering of the $\mathrm{GaN}$ decomposition temperature was thereby predicted. The corrected decomposition temperature can guide the practical maximum annealing temperature of $\mathrm{GaN}$ thin films.

\section{Experimental}

GaN epilayers were grown by metalorganic chemical vapor deposition (MOCVD) and a novel growth method that combines hydride vapor phase epitaxy with MOCVD (H-MOVPE). For MOCVD growth TEGa (triethylgallium) and $\mathrm{NH}_{3}$ were used as source in a $\mathrm{N}_{2}$ carrier gas, and the films were grown at $850^{\circ} \mathrm{C}$, a reactor pressure of 76 Torr, and an inlet N/Ga molar ratio of $\sim 3000$. The thickness of MOCVD grown $\mathrm{GaN}$ was measured by cross-sectional SEM to be $\sim 1.0 \mu \mathrm{m}$. H-MOVPE is an approach that reacts a organometallic (trimethylgallium in this study) with $\mathrm{HCl}$ to supply volatile $\mathrm{GaCl}$, which then reacts with $\mathrm{NH}_{3}$ downstream to form GaN. The detailed description of H-MOVPE and its operation conditions is given elsewhere [10]. The samples for this study were grown at $900{ }^{\circ} \mathrm{C}$, atmospheric pressure, and an inlet $\mathrm{N} / \mathrm{Ga}$ ratio of 125 . The $\mathrm{HCl} / \mathrm{Ga}$ ratio was set to 2 to provide optimum film quality and scavenge gas phase metallic residuals. The thickness of H-MOVPE grown GaN was also measured by cross-sectional SEM and it was $\sim 3.2 \mu \mathrm{m}$.

The depth-dependent Raman measurements of MOCVD and H-MOVPE deposited GaN films were made using a Renishaw System-2000 in confocal mode. The laser output power was set at $20 \mathrm{~mW}$ with an excitation wavelength of $514.5 \mathrm{~nm}$. The focused beam size was approximately $2 \mu \mathrm{m}$, accumulation time was $10 \mathrm{sec}$, and a CCD detector was employed. By controlling the slit width and CCD pixel size, the signals from different depth could be separated.

\section{Theoretical}

The relation between the film stress and substrate curvature $(\kappa)$ is well known and given by Stoney's equation, $\kappa=6 \sigma_{f} h_{f} /\left(M_{s} h_{s}^{2}\right)$ [7]. In this expression, $\sigma_{f}$ is the biaxial stress in the film, $h_{f}, h_{s}$ are the thicknesses of the film $(3.2 \mu \mathrm{m}$ : H-MOVPE, $1.0 \mu \mathrm{m}$ : MOCVD) and substrate $(300 \mu \mathrm{m}$ : sapphire), respectively. $M_{s}$ is the substrate biaxial modulus, which is $602 \mathrm{GPa}$ for sapphire [11].

Shen et al. [9] recently developed equations for hexagonal GaN eptiaxial thin films assuming isotropic strain of epitaxial film. These equations predict the changes in strain energy of the film with respect to the growth plane for the case when the planes of film growth and the underlying substrate are both hexagonal. Substituting and rearranging the original equation gives:

$$
a(\AA)=a_{0}-a_{0}\left\{\left(s_{11}+s_{12}\right) E_{s}\right\}^{0.5} .
$$

The range in the reported values for the elastic compliances in Eq. (1) are $s_{11}=0.00297$ to 0.00502 and $s_{12}$ $=-0.00066$ to $-0.00121 \mathrm{GPa}^{-1}[6,12-14]$. In this work the range-centered values $s_{11}=0.003995$ and $s_{12}=$ -0.000935 were selected. The values of $a_{0}$ are the unstrained GaN lattice parameters (3.189 $\AA$ for MOCVD and $3.067 \AA$ for H-MOVPE films) and $a$ is the lattice parameter of the strained GaN epilayers. 
Frank-van der Merwe's thick overgrowth model was used to estimate the MOCVD grown GaN/sapphire interface shear modulus. The total strain energy per atom, $E_{s}$, of epilayer containing misfit dislocations and periodic elastic strains, is expressed as [9]:

$$
E_{s}=\frac{G_{i} c^{2}}{4 \pi^{2} d}[\underbrace{1+\lambda-\sqrt{1+\lambda^{2}}}_{E_{i}=\text { Interface Energy }}-\underbrace{\left\{1+\frac{G_{b}\left(1-v_{a}\right)}{G_{a}\left(1-v_{b}\right)}\right\}^{-1} \lambda \ln \left(2 \lambda \sqrt{1+\lambda^{2}}-2 \lambda^{2}\right)}_{E_{e}^{b}=\text { Strain Energy in the Film }}-\underbrace{\left\{1+\frac{G_{a}\left(1-v_{b}\right)}{G_{b}\left(1-v_{a}\right)}\right\}^{-1} \lambda \ln \left(2 \lambda \sqrt{1+\lambda^{2}}-2 \lambda^{2}\right)}_{E_{e}^{a}=\text { Strain Energy in the Substrate }}] \text {, }
$$

where $\lambda=\frac{4 \pi}{G_{i}}\left\{\frac{G_{a} G_{b}}{G_{a}\left(1-v_{b}\right)+G_{b}\left(1-v_{a}\right)}\right\}\left(\frac{b-a}{b+a}\right)$ is misfit, $G_{i}$ is the shear modulus at the interface, $d \approx c$ is the separation of the atoms of the crystal planes, and $c=2 a b /(a+b)$. The lattice parameters of substrate and film are $a$ and $b$, respectively. $G_{a}$ and $G_{b}$ are the shear moduli of substrate and film and $v_{a}$ and $v_{b}$ are Poisson's ratio for the substrate and film, respectively.

\section{Results and discussion}

Fig. 1 shows the measured Raman shifts of GaN $E_{2}$ mode of MOCVD and H-MOVPE grown epilayers at varying depths from the surface. In the case of MOCVD grown films, the $\mathrm{E}_{2}$ peak was found to vary from $568 \mathrm{~cm}^{-1}$ (GaN surface) to $569 \mathrm{~cm}^{-1}$ (GaN/sapphire interface). In the case of H-MOVPE grown samples, however, the $\mathrm{E}_{2}$ peak varied from $570 \mathrm{~cm}^{-1}$ (GaN surface) to $575 \mathrm{~cm}^{-1}$ (GaN/sapphire interface). This indicates that the surface of H-MOVPE grown epilayer was not free from hydrostatic strain, whereas MOCVD grown film contained no residual strain at the surface.

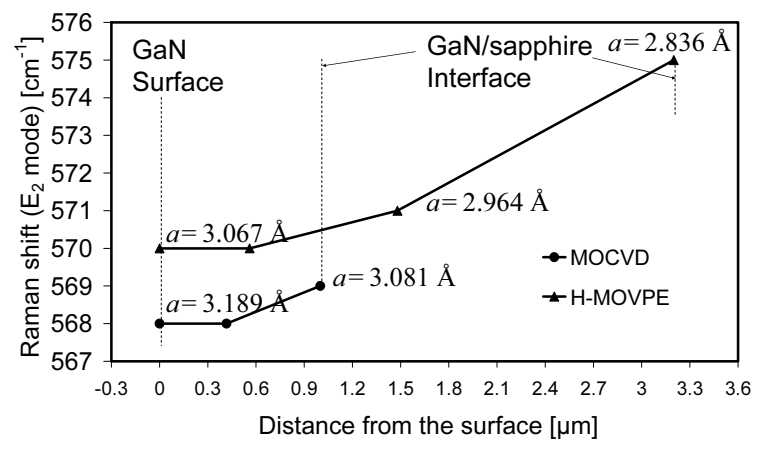

Fig. 1 Raman shift ( $E_{2}$ mode) depth profile of MOCVD and H-MOVPE grown GaN layers.

It is known that a biaxial stress of $1 \mathrm{GPa}$ shifts the $\mathrm{E}_{2}$ Raman mode by $2.7 \pm 0.3 \mathrm{~cm}^{-1}$ [4], and a hydrostatic stress of $1 \mathrm{GPa}$ shifts it by $4.17 \mathrm{~cm}^{-1}$ [15], thus these values were used to calculate strain energy of the epilayer. The hydrostatic component of the strain energy was neglected when calculating the strain energy of the MOCVD sample. The H-MOVPE grown sample, however, contained sufficient impurity so the hydrostatic component was counted. The curvature radius of the sapphire substrate at the thick HMOVPE film/sapphire interface was estimated to be $1.5 \mathrm{~m}$ and thus substrate bowing is expected by the Stoney's equation. The calculated results by Eq.(1) for H-MOVPE sample are tabulated in Table 1.

Table 1 Strain energy and lattice parameter of the H-MOVPE sample with depth

\begin{tabular}{lll}
\hline Depth from the surface $[\mu \mathrm{m}]$ & Strain energy $[\mathrm{GPa}]$ & Lattice parameter $[\AA]$ \\
\hline $0-0.6$ & 0.48 (hydrostatic) & $3.067 \pm 0.030$ \\
1.5 & 0 (biaxial) & \\
3.2 (interface) & 0.37 (biaxial) & $2.964 \pm 0.025$ \\
\hline
\end{tabular}


For the MOCVD sample, the strain seems to release quickly, so only interface was measured to contain residual stress $(0.37 \mathrm{GPa})$. Frank-van der Merwe's thick overgrowth model was used to calculate the interface shear modulus, since the stress was small near the interface and the surface was strain-free. The value of $G_{i}$ can be calculated from Eq. (3), as the total strain energy of the interface is measured by Raman peak shift. In this system, the values $G_{a}=1.38 \times 10^{11} \mathrm{~Pa}, G_{b}=1.01 \times 10^{11} \mathrm{~Pa}, v_{a}=0.2224, v_{b}=0.2578$ [12], $a=2.747 \AA, b=3.189 \AA$, and $E_{s}=0.37 \mathrm{GPa}$ were used to calculate the interface shear modulus of MOCVD sample. The estimated $G_{i}$ is $0.14 \times 10^{11} \mathrm{~Pa}$ which implies that the MOCVD interface is much less rigid than the overlying $\mathrm{GaN}$ layer and the sapphire substrate.

The measured strain energy of MOCVD grown GaN/sapphire interface $(0.37 \mathrm{GPa}=5.0 \mathrm{~kJ} / \mathrm{mole})$ was used to correct the formation Gibbs energy of $\mathrm{GaN}$, and the decomposition temperature of GaN was calculated using the ThermoCalc Software. The thermochemical data of unstrained GaN were taken from the work of Davydov et al. [16]. The decomposition temperature of MOCVD GaN with residual strain was found to be $1011 \mathrm{~K}$ at the interface, which is lower than the unstrained GaN (1055 K). Kumar et al. [17] observed that the GaN/sapphire interface begins to be heavily damaged on annealing the substrate, which suggests that the decomposition initiates at the interface. The result of this study agrees qualitatively with Kumar's observation.

\section{Summary}

Strain energy in the GaN epilayer on sapphire was measured by micro-Raman Spectroscopy. Depth profiling in confocal mode was demonstrated to be a versatile and non-destructive approach for depth dependent stress and curvature measurement. The strain energy at MOCVD grown GaN/sapphire interface was estimated to be $5.0 \mathrm{~kJ} / \mathrm{mole}$. Significant curvature was calculated by Stoney's equation for HMOVPE sample. Shen's approach was utilized to calculate lattice constants of strained films, and Frankvan der Merwe's thick overgrowth model was used to calculate the shear modulus of the MOCVD grown GaN/sapphire interface. Finally the decomposition temperature of GaN was estimated and temperature lowering was predicted at the GaN/sapphire interface.

\section{References}

[1] S. Strite and H. Morkoç, J. Vac. Sci. Technol. B 10, 1237 (1992).

[2] Yu.V. Melnik, K.V. Vassilevski, I.P. Nikitina, A.I. Babanin, V. Yu. Davydov, and V.A. Dmitriev, MRS Internet J. Nitride Semicond. Res. 2, 39 (1997).

[3] A. Tabata, R. Enderlein, J. R. Leite, S.W. da Silva, J.C. Galzerani, D. Schikora, M. Kloidt, and K. Lischka, J. Appl. Phys. 79, 4137 (1996).

[4] V. Yu. Davydov, N. S. Averkiev, I. N. Goncharuk, D. K. Nelson, I. P. Nikitina, A. S. Polkovnikov, A. N. Smirnov, M. A. Jacobson, and O. K. Semchinova, J. Appl. Phys. 82, 5097 (1997).

[5] J. Ager, T. Suski, S. Ruvinov, J. Krueger, G. Conti, E. Weber, M. Bremser, R. Davis, and C. Kuo, Mater. Res. Soc. Symp. Proc. 449, 775 (1997).

[6] V. Yu. Davydov, Yu. E. Kitaev, I. N. Goncharuk, A. N. Smirnov, J. Graul, O. Semchinova, D. Uffmann, M. B. Smirnov, A. P. Mirgorodsky, and R. A. Evarestov, Phys. Rev. B 58, 12899 (1998).

[7] G. Stoney, Proc. R. Soc. Lond. A 82, 172 (1909).

[8] J. Shen, S. Johnston, S. Shang, and T. Anderson, J. Cryst. Growth, 240, 6 (2002).

[9] J. H. van der Merwe, Proc. Phys. Soc. Lond. A 63, 616 (1950).

[10] O. Kryliouk, M. Reed, T. Dann, T. J. Anderson, and B. Chai, Mater. Sci. Eng. B 66, 26 (1999).

[11] Landolt-Börnstien, Numerical Data and Functional Relationships in Science and Technology, edited by K. H. Hellwege, A. M. Hellwege, and D. F. Nelson (Springer, Berlin, 1979, 1992), Vols. III:11, III:29.

[12] Y. Takagi, M. Ahart, T. Azuhata, T. Sota, K. Suzuki, and S. Nakamura, Physica B 219-220, 547 (1996).

[13] A. Polian, M. Grimsditch, and I. Grzegory, J. Appl. Phys. 79, 3343 (1996).

[14] V. A. Savastenko and A. U. Sheleg, phys. stat. sol. (a) 48, K135 (1978).

[15] P. Perlin, C. J. Carrilon, J. P. Itie, A. S. Miguel, I. Grzegory, and A. Polian, Phys. Rev. B 45, 83 (1992)

[16] A.V. Davydov, W.J. Boettinger, U.R. Kattner, and T.J. Anderson, phys. stat. sol. (a) 188, 407 (2001).

[17] M. Senthil Kumar, G. Sonia, V. Ramakrishnan, R. Dhanasekaran, and J. Kumar, Physica B 324, 223 (2002). 